\title{
Using Biomarkers for Acute Kidney Injury: Barriers and Solutions
}

\author{
Zoltán H. Endre \\ Department of Nephrology, Prince of Wales Hospital, and Prince of Wales Clinical School, University of \\ New South Wales, Sydney, N.S.W., and The School of Medicine, University of Queensland, Brisbane, Qld., Australia; \\ Department of Medicine, University of Otago, Christchurch, New Zealand
}

\section{Key Words}

Biomarker · Acute kidney injury · Cutoff

\begin{abstract}
The clinical implementation of urinary and plasma renal injury biomarkers has been hampered by the variability associated with nonstandardized commercially available biomarker assays, uncertainty and variations in patient selection criteria, and the absence of context-specific cutoffs for biomarker concentrations. These limitations are increased by comparison with serum creatinine to define acute kidney injury. The critical problem affecting biomarker performance is patient heterogeneity involving the cause, context (including comorbidity and baseline renal function), and timing of the injury. We suggest strategies for stratifying subjects to provide appropriate context, and illustrate a creatinine-independent method for defining thresholds for biomarker concentrations in these contexts which utilizes the same sensitivity for the clinical outcomes of dialysis or death. Large multicenter cohort studies are needed to validate the proposed cutoffs.

(c) 2014 S. Karger AG, Basel
\end{abstract}

Changing Paradigms in Acute Kidney Injury: From Mechanisms to Management - Proceedings of the 5th Annual UAB-UCSD O'Brien Center Symposium (San Diego, Calif., USA, March 4, 2014).

\section{KARGER}

E-Mail karger@karger.com www.karger.com/nec

\section{Introduction}

Novel biomarkers outperform serum creatinine in the early diagnosis of acute kidney injury (AKI). Despite widespread enthusiasm from nephrologists, intensivists, and cardiologists, the implementation of urinary and plasma renal injury biomarkers has been hampered by the variability associated with nonstandardized commercially available biomarker assays, uncertainty and variations in patient selection criteria, and the absence of context-specific cutoffs for biomarker concentrations. Fundamental to these concerns is the absence of a genuine gold standard against which to evaluate these cutoffs, since comparison with creatinine represents a circular argument if creatinine is also used to define the outcome. Despite such limitations, the Acute Dialysis Quality Initiative (ADQI) has recommended redefining AKI diagnosis and staging in terms of both damage and functional markers $[1,2]$. We have suggested strategies for stratifying subjects to provide an appropriate context and methods of defining thresholds for biomarker concentrations in these contexts. Large multicenter cohorts will be needed to validate the proposed cutoffs. The critical problem affecting biomarker performance is patient heterogeneity involving the cause, context (including comorbidity and baseline renal function), and timing of the injury. Solutions to these barriers are discussed below.
Prof. Zoltán H. Endre

Department of Nephrology, Prince of Wales Hospital High Street, Randwick

Sydney, NSW 2031 (Australia)

E-Mail z.endre@unsw.edu.au 
As we have highlighted previously [3], the initial biomarker performance is usually outstanding because the biomarkers are selected on the basis of relatively homogeneous cohorts that differ only in terms of the presence or absence of AKI. The biomarkers are selected after receiver operating characteristic analysis yields an area under the curve (AUC) well above 0.90. Such preliminary studies are followed by similarly carefully selected homogenous validation cohorts with similarly excellent biomarker performance results. However, then the biomarker is applied to less homogenous patient groups in necessarily more heterogeneous cohorts (so-called phase 3 studies), and when these include high-risk patients as well as affected patients the biomarker performance is automatically degraded. In many studies, the outstanding performance of novel biomarkers with initial AUCs above 0.90 becomes of questionable utility, with AUCs frequently below 0.70 . Examples of performance degradation abound not only for older biomarkers such as tubular enzymuria, e.g. $\gamma$-transpeptidase [4] versus the results of Endre et al. [5], but also for novel biomarkers, e.g. neutrophil gelatinase-associated lipocalin (NGAL) and liver fatty acid-binding lipoprotein [6] versus the results of Siew et al. [7].

The critical factor in the degradation of biomarker performance is heterogeneity among the patients undergoing assessment [3]. This heterogeneity may involve the duration of renal injury prior to biomarker measurement or of baseline renal function prior to injury. Stratification by either or preferably both of these parameters improves biomarker performance [5]. Heterogeneity may involve the cause of AKI (e.g. very diverse causes such as sepsis vs. ischemia-reperfusion injury) [3]. Heterogeneity of the cause may simply involve the inclusion of mild (low-risk) versus moderate-to-severe (high-risk) injury secondary to the same cause, such as so-called 'prerenal' versus established (intrinsic) hypoperfusion AKI [8], where what is actually a continuum of injury appears discontinuous because of differing selection criteria such as early reversibility or response to fluid therapy, although these criteria really just represent severity criteria [9]. Heterogeneity of comorbidity may similarly play a role in degrading the performance of individual biomarkers. This may be the explanation for the differing performance of urinary versus plasma NGAL in differing contexts. For example, among adults and children immediately after cardiopulmonary bypass surgery, plasma but not urinary NGAL was predictive of AKI in the adult population [10] while urinary but not plasma NGAL was predictive in children [11]. Nevertheless, in both populations the AUCs were

Using Biomarkers for AKI: Barriers and Solutions modest (in the mid 0.70 range), and quintile comparisons rather than preselected cutoffs were used to draw conclusions about performance.

So, what strategies can we use to improve biomarker performance? Careful patient selection, timed selection of specific biomarkers, and serial biomarker measurement, perhaps in a panel of different biomarkers, are useful future strategies.

Careful patient selection for some of these parameters will prospectively improve the biomarker performance. Provided the biomarker time course is known, it is theoretically possible to select ideal biomarker(s) when the injury is reasonably well timed. Thus, the immediate answer in cardiopulmonary bypass appears obvious from the example above. However, even this context remains inadequately defined since significant biomarker dilution is likely in the immediate postbypass setting. An analogous setting is cardiac arrest. We combined creatinine and volume kinetics to account for fluid type, and rates of fluid infusion and urine output to analyze the effect of dilution on biomarker (serum creatinine) profiles in 49 patients after cardiac arrest (mostly out-of-hospital arrest) [12]. A reduction in creatinine production was required to account for the degree of decrease in serum creatinine observed in the majority of patients in this setting. More importantly, only a decrease in the glomerular filtration rate (GFR) of approximately $50 \%$ could account for maintenance of a normal serum creatinine in 6 patients, while an immediate and progressive increase was only observed in 2 patients. These results strongly support the use of fluid balance to correct serum creatinine and avoid a misdiagnosis of AKI, as advocated by Macedo et al. [13]. There is no reason to assume that other plasma biomarkers, including NGAL, are not similarly diluted. We would also advocate for correction for dilution of both functional and structural biomarkers in the analysis of postoperative settings associated with significant fluid therapy.

Thus, timing and correction for dilution may enhance the biomarker performance prospectively. The optimal timing is also modified by baseline renal function, as demonstrated in the EARLYARF study where the peak performance of each biomarker varied with both the duration after renal injury and the baseline GFR before renal injury [5]. While the stratification used to demonstrate performance enhancement in that study was used in retrospect, the message for prospective use is to measure the biomarkers at specific time points after injury based on both the individual biomarker profile and the baseline GFR. Such an approach lends itself to the identification 
Table 1. Urinary biomarker cutoffs based on sensitivity linked to dialysis or death

\begin{tabular}{|c|c|c|c|c|c|c|}
\hline & $\begin{array}{l}\text { NGAL, } \\
\mathrm{ng} / \mathrm{ml}\end{array}$ & $\begin{array}{l}\text { KIM-1, } \\
\mathrm{pg} / \mathrm{ml}\end{array}$ & $\begin{array}{l}\text { IL-18, } \\
\mathrm{pg} / \mathrm{ml}\end{array}$ & $\begin{array}{l}\text { CysC, } \\
\mathrm{ng} / \mathrm{ml}\end{array}$ & $\begin{array}{l}\mathrm{sCr}, \\
\mathrm{mg} / \mathrm{dl}\end{array}$ & $\mathrm{n}$ \\
\hline \multicolumn{7}{|l|}{ Sensitivity-linked method } \\
\hline $62 \%$ sensitivity for death or RRT & 140 & 1,850 & 118 & 950 & $>1.3 \mathrm{mg} / \mathrm{dl}$ or $>50 \% *$ & $507[18]$ \\
\hline \multicolumn{7}{|c|}{ Emergency room screening } \\
\hline Cutoff & 104 & 2,817 & 65 & 171 & 1.4 & \\
\hline Sensitivity/specificity for AKI & $68 / 81$ & $52 / 79$ & $43 / 58$ & $46 / 59$ & $81 / 82$ & $1,635[20]$ \\
\hline
\end{tabular}

of a panel of biomarkers that includes one or more that increase early and others that increase later after injury. This would allow timing of the injury as well as diagnosis. An alternative strategy with a single biomarker is serial measurement. This is analogous to serial troponin measurement in the diagnosis of acute myocardial ischemic injury. At present, biomarker profiles are not well defined. However, several studies suggest that a reduction in the concentration of some injury biomarkers predicts recovery; for example, a reduction in urinary NGAL and hepatocyte growth factor on day 14 in the ATN trial predicted survival and dialysis independence at 60 days [14]. While encouraging, few serial measurement studies of urinary or plasma injury biomarkers have been performed that allow comment on clinical utility in the prediction of recovery.

\section{Can We Select Better Biomarkers?}

While maneuvers that enrich the population homogeneity yield higher biomarker AUC values for diagnosis, population enrichment is not always an option. Common clinical scenarios are examples of this. For example, heterogeneous populations are the norm in the emergency department or on admission to adult intensive care units. Thus, a realistic context is critical. The newest novel biomarkers are the cell cycle arrest markers urinary insulinlike growth factor-binding protein 7 (IGFBP7) and tissue inhibitor of metalloproteinases-2 (TIMP-2), both inducers of $\mathrm{G} 1$ cell cycle arrest, a key mechanism implicated in AKI. In a heterogeneous cohort of 728 critically ill patients, the combination of these two biomarkers demonstrated an AUC of 0.80 ( 0.76 and 0.79 alone) [15]. A headto-head comparison with all the other novel biomarkers currently available revealed that urinary [TIMP-2] . [IGFBP7] was significantly superior to all previously de- scribed markers of AKI $(p<0.002)$, none of which achieved an AUC above 0.72. The authors also demonstrated the added value of the biomarkers by showing that [TIMP-2] · [IGFBP7] significantly improved the risk stratification when added to a 9-variable clinical model analyzed using the Cox proportional hazards model, a generalized estimation equation, and the integrated discrimination improvement or net reclassification improvement strategies. In sensitivity analyses, [TIMP-2] . [IGFBP7] remained significant and superior to all other markers regardless of changes in the reference creatinine method. While these results are exciting by any reckoning, the strategy underpinning the improved biomarker performance may be that these 2 cell cycle inhibitors were selected from over 300 biomarkers in 3 distinct cohorts including patients with sepsis, shock, major surgery, and trauma. Thus, the selection was based on performance in a heterogeneous discovery cohort rather than a homogeneous one. Presumably, this strategy created a higher initial hurdle for selection but then resulted in less degradation of the biomarker performance when tested in other similar real-world heterogeneous settings.

\section{Patient Classification Using Biomarkers Requires Reliable Cutoffs}

Up to now, these have been validated against serum creatinine since absolute or relative increases in creatinine are the primary basis for the consensus definitions of AKI, although the changes actually are surrogates for change in GFR [16] even when incorrectly calculated [17]. Perhaps the real reason for using creatinine is our conceptualization of the GFR as an integrated marker of renal function which has superseded urea (or BUN) as a means of identifying the need for dialysis. In any case, validation of biomarker performance against creatinine- 
based definitions creates a circular argument. While a biomarker increase can predict an increase in creatinine, only the inevitably delayed change in creatinine can diagnose AKI. This further ignores that creatinine is a marker of function, and actually a continuous variable, while injury biomarkers detect structural damage, which is arguably a dichotomous variable (present or absent). This also ignores the frequently recited deficiencies of serum creatinine [16]. Thus for validity, the cutoffs for functional and damage markers in the ADQI AKI matrix need to be derived against an independent gold standard. Unlike preclinical studies, renal histology is not routinely available as the gold standard. Since dialysis requirement and death are significant clinical outcomes of AKI, we proposed a method for establishing biomarker cutoffs by selecting values that had the same sensitivity for these outcomes as serum creatinine [18]. This method was examined in a discovery cohort $(\mathrm{n}=253)$ and then assessed in a validation cohort $(n=254)$. The method generated values (table 1) for absolute urinary biomarker concentrations that were similar to those revealed for NGAL by a meta-analysis of observational studies in cardiorenal AKI [19] and for both NGAL and KIM-1 in a large cohort screened in the emergency department [20]. While the absolute values require validation in large cohorts, the method is robust and will allow classification, staging, and differential diagnosis of AKI using the damage function matrix recommended by the ADQI. This or similar approaches that define cutoffs independently of serum creatinine will allow prospective validation of the biomarker-positive, creatinine-negative category of AKI which is needed for application of the ADQI proposals and will finally allow evolution to injury biomarker-based triaging of patients outside of the domain of clinical research.

\section{Conclusions}

- Biomarker performance is critically affected by heterogeneity of the cause, context, baseline renal function, and timing of the injury.

- Biomarker cutoffs need to be assessed against outcomes independently of serum creatinine.

- Sensitivity for dialysis or death can be used to identify biomarker cutoffs for AKI diagnosis.

\section{References}

-1 Murray PT, Mehta RL, Shaw A, Ronco C, Endre Z, Kellum JA, Chawla LS, Cruz D, Ince C, Okusa MD, the F: Current use of biomarkers in acute kidney injury: report and summary of recommendations from the 10th Acute Dialysis Quality Initiative consensus conference. Kidney Int 2013;1-9.

-2 Endre ZH, Kellum JA, Di Somma S, Doi K, Goldstein SL, Koyner JL, Macedo E, Mehta RL, Murray PT: Differential diagnosis of AKI in clinical practice by functional and damage biomarkers: workgroup statements from the tenth Acute Dialysis Quality Initiative Consensus Conference. Contrib Nephrol 2013; 182:30-44.

$\checkmark 3$ Endre $\mathrm{ZH}$, Pickering JW: Biomarkers and creatinine in AKI: the trough of disillusionment or the slope of enlightenment? Kidney Int 2013;84:644-647.

4 Westhuyzen J, Endre Z, Reece G, Reith D, Saltissi D, Morgan T: Measurement of tubular enzymuria facilitates early detection of acute renal impairment in the intensive care unit. Nephrol Dial Transpl 2003;18:543-551.
-5 Endre ZH, Pickering JW, Walker RJ, Devarajan P, Edelstein CL, Bonventre JV, Frampton CM, Bennett MR, Ma Q, Sabbisetti VS, Vaidya VS, Walcher AM, Shaw GM, Henderson SJ, Nejat M, Schollum JBW, George PM: Improved performance of urinary biomarkers of acute kidney injury in the critically ill by stratification for injury duration and baseline renal function. Kidney Int 2011;79:1119-1130.

-6 Mishra J, Dent C, Tarabishi R, Mitsnefes MM, Ma Q, Kelly C, Ruff SM, Zahedi K, Shao M, Bean J, Mori K, Barasch J, Devarajan P: Neutrophil gelatinase-associated lipocalin (NGAL) as a biomarker for acute renal injury after cardiac surgery. Lancet 2005;365:1231-1238.

$\checkmark 7$ Siew ED, Ware LB, Bian A, Shintani A, Eden SK, Wickersham N, Cripps B, Ikizler TA: Distinct injury markers for the early detection and prognosis of incident AKI in critically ill adults with preserved kidney function. Kidney Int 2013;84:786-794.

-8 Parikh CR, Han G: Variation in performance of kidney injury biomarkers due to cause of acute kidney injury. Am J Kidney Dis 2013;62: 1023-1026.

-9 Nejat M, Pickering JW, Devarajan P, Bonventre JV, Edelstein CL, Walker RJ, Endre ZH: Some biomarkers of acute kidney injury are increased in pre-renal acute injury. Kidney Int 2012;81:1254-1262.
10 Parikh CR, Devarajan P, Zappitelli M, Sint K, Thiessen-Philbrook H, Li S, Kim RW, Koyner JL, Coca SG, Edelstein CL, Shlipak MG, Garg AX, Krawczeski CD; TRIBE-AKI Consortium: Postoperative biomarkers predict acute kidney injury and poor outcomes after adult cardiac surgery. J Am Soc Nephrol 2011;22: 1748-1757.

11 Parikh CR, Devarajan P, Zappitelli M, Sint K, Thiessen-Philbrook H, Li S, Kim RW, Koyner JL, Coca SG, Edelstein CL, Shlipak MG, Garg AX, Krawczeski CD; TRIBE-AKI Consortium: Postoperative biomarkers predict acute kidney injury and poor outcomes after pediatric cardiac surgery. J Am Soc Nephrol 2011; 22:1737-1747.

12 Pickering JW, Ralib AM, Endre ZH: Combining creatinine and volume kinetics identifies missed cases of acute kidney injury following cardiac arrest. Crit Care 2013;17:R7.

-13 Macedo E, Bouchard J, Soroko SH, Chertow GM, Himmelfarb J, Ikizker TA, Paganini EP, Mehta RL: Fluid accumulation, recognition and staging of acute kidney injury in critically-ill patients. Crit Care 2010;14:R82. 
14 Srisawat N, Wen X, Lee M, Kong L, Elder M, Carter M, Unruh M, Finkel K, Vijayan A, Ramkumar M, Paganini E, Singbartl K, Palevsky PM, Kellum JA: Urinary biomarkers and renal recovery in critically ill patients with renal support. Clin J Am Soc Nephrol 2011;6:1815-1823.

15 Kashani K, Al-Khafaji A, Ardiles T, Artigas A, Bagshaw SM, Bell M, Bihorac A, Birkhahn R, Cely CM, Chawla LS, Davison DL, Feldkamp T, Forni LG, Gong MN, Gunnerson KJ, Haase M, Hackett J, Honore PM, Hoste EA, JoannesBoyau O, Joannidis M, Kim P, Koyner JL, Laskowitz DT, Lissauer ME, Marx G, McCullough PA, Mullaney S, Ostermann M, Rimmele T, Shapiro NI, Shaw AD, Shi J, Sprague AM, Vincent J-L, Vinsonneau C, Wagner L, Walker MG, Wilkerson RG, Zach- arowski K, Kellum JA: Discovery and validation of cell cycle arrest biomarkers in human acute kidney injury. Crit Care 2013;17:R25.

16 Endre ZH, Pickering JW, Walker RJ: Clearance and beyond: the complementary roles of GFR measurement and injury biomarkers in acute kidney injury (AKI). Am J Physiol Renal Physiol 2011;301:F697-F707.

17 Pickering J, Endre ZH: GFR shot by RIFLE: errors in staging acute kidney injury. Lancet 2009;373:1318-1319.

18 Pickering JW, Endre ZH: Linking injury to outcome in acute kidney injury: a matter of sensitivity. PLoS One 2013;8:e62691.

19 Haase M, Devarajan P, Haase-Fielitz A, Bellomo R, Cruz DN, Wagener G, Krawczeski CD, Koyner JL, Murray P, Zappitelli M, Goldstein SL, Makris K, Ronco C, Martensson J,
Martling C-R, Venge P, Siew E, Ware LB, Ikizler TA, Mertens PR: The outcome of neutrophil gelatinase-associated lipocalin-positive subclinical acute kidney injury a multicenter pooled analysis of prospective studies. J Am Coll Cardiol 2011;57:1752-1761.

20 Nickolas TL, Schmidt-Ott KM, Canetta P, Forster C, Singer E, Sise M, Elger A, Maarouf O, Sola-Del Valle DA, O'Rourke M, Sherman E, Lee P, Geara A, Imus P, Guddati A, Polland A, Rahman W, Elitok S, Malik N, Giglio J, ElSayegh S, Devarajan P, Hebbar S, Saggi SJ, Hahn B, Kettritz R, Luft FC, Barasch J: Diagnostic and prognostic stratification in the emergency department using urinary biomarkers of nephron damage: a multicenter prospective cohort study. J Am Coll Cardiol 2012;59:246-255. 